\title{
Ground, Warmth, and Light: Facilitating Conditions for Reflection and Transformative Dialogue
}

\author{
Kaisu Mälkki (Corresponding author) \\ Research Group of Educational Psychology, Department of Teacher Education \\ P.O. Box 9, Siltavuorenpenger 5A, University of Helsinki, Helsinki 00014, Finland \\ Tel: 358-294-129-795Ｅ-mail: kaisu.malkki@helsinki.fi
}

\section{Larry Green}

City University of Seattle, Vancouver Campus

816 Millbank, Vancouver, BC V5Z 3Z4, Canada

Tel: 1-778-233-0377 E-mail: genero@telus.net

Received: August 25, 2016 Accepted: October 15, 2016 Published: October 26, 2016

doi:10.5296/jei.v2i2.9947ＵRL: http://dx.doi.org/10.5296/jei.v2i2.9947

\begin{abstract}
In this paper we look into the conditions in which dialogue could be utilized to facilitate transformative learning and reflection. We explore the notion of a safe and accepting learning environment from the relational and phenomenological viewpoint, and analyze what it actually means and how it may be developed. We understand facilitating conditions as an inseparable aspect of the learning process similarly to the way a greenhouse supplies right conditions to facilitate the growth of the plant. Similarly as the ground, warmth and light play their essential roles in the growing of the plant, in our paper we offer conceptual tools to understand the dynamics of safe and accepting learning environment in facilitating the processes of reflection and transformative learning.
\end{abstract}

Keywords: transformative learning, dialogue, facilitation, reflection, learning environment, pedagogy, teaching

\section{Introduction}

In this paper we look into the conditions in which dialogue could be utilized to facilitate 
transformative learning and reflection. We explore the notion of a safe and accepting learning environment from the relational and phenomenological viewpoint, and analyze what it actually means and how it may be developed. We understand facilitating conditions as an inseparable aspect of the learning process similarly to the way a greenhouse supplies right conditions to facilitate the growth of the plant. Similarly as the ground, warmth and light play their essential roles in the growing of the plant, in our paper we offer conceptual tools to understand the dynamics of safe and accepting learning environment in facilitating the processes of reflection and transformative learning.

The role of dialogue in Mezirow's transfórmative learning theory is explicitly emphasized as a venue for exploring alternative viewpoints which in turn stimulates reflective thinking. Dialogue can be understood as a conversation between two subjects rather than between subject and object (see Hooks, 2007; Freire \& Shor, 1989; Rainio, 2010). Similar humanizing and respectful characteristics are attributed to dialogue by Mezirow (1991), who follows Habermas' ideas on the ideal speech situation. In addition Mezirow claims that participants experience increased autonomy as a result of this process. These fine sentences express ideal outcomes of an educational activity, rather than processes or methods that might actualize them in pedagogical praxis (see also Illeris, 2007). In a more realistic tone Mezirow (2000) notes that dialogue is subject to human error and does not necessarily make issues of power, gender, class, for example, subject to critical thinking and reflection. Mezirow (1991, 2000) calls for a safe and accepting learning environment as essential for the kind of dialogue that promotes reflection and transformative learning. Again, this is left as an ideal generalization without specifying how it stimulates reflective thinking nor what must be done to promote such an environment.

In this paper we explore the processes by which a safe and accepting learning environment can be generated. Safety is here understood as a relative term. That is, there is no situation that is absolutely safe except being dead. Thus, the risk factor is a necessary aspect to be considered in reaching understanding of the safety of the environment. We look into it from a relational and phenomenological viewpoint: relational in the sense that we consider the safe and accepting learning environment to be formed in the interaction among the participants; and phenomenological in the sense that we consider the issue with conceptualizations that aim to grasp the first-person experiential viewpoint. We employ the concept of reflection to refer to the process of becoming aware of, and then interrogating the assumptions governing our thinking, feeling and acting (Mezirow, 1991; Mälkki, 2011). Thus, reflection is here understood as a specific kind of critical thinking and assessment of premises, that which is oriented internally, towards one's own meaning perspectives that, consequently, orient understanding and interpretation also concerning external knowledge. More specifically, we define reflection as the process by which we give form to, and thereby become aware of, our prereflective expectations. It is, therefore, internally or self-focused rather than externally, or world focused. It presupposes that a person is aware that their reality is filtered through their concepts, beliefs, and meanings. We tend to see what we expect to see. That is, until we discover that those assumptions can't produce the results that we intend. It is at that point that reflection becomes a possibility. We say possibility because there is always the option, for 
example, to blame the other and thereby preserve one's premises (Mälkki, 2011). If, however, the person chooses and is able to reflect on and critically inspect one's premises, then there is a possibility to revise those premises in favor of premises that include and account for the event that escaped one's predictions. Further, while our study is grounded in the research tradition of adult learning, we believe that this is a capacity that can be taught and learned, rather than depending on biological age, as such. That is, school age children can reflect on their unmet expectations with the support of an adult be it a teacher or a parent. By 'walking' the child through the reflective process, the child begins to internalize the procedure and by adolescence should be capable of making the choice between reflection or blame (external attribution).

The paper is organized as follows. Firstly, we review the purpose or function of reflection in Mezirow's framework. Secondly, we submit the notion of "safe and accepting learning environment in facilitating reflection" to a conceptual analysis that reveals its underlying assumptions and thereby, open up questions for further analysis. Thirdly, we consider the challenges and prerequisites implicated in reflection as factors to be considered in the design of such an environment. We draw on previously published work, explicitly rooted in Mezirow's theory, on the relationship between reflection and edge-emotions, indicative of the challenges to reflection (Mälkki, 2010, 2011; see also Mälkki \& Green, 2014; Mälkki \& Green, 2016). Fourthly, we consider how the factors that produce the challenge of working with "edge-emotions" can be understood. Once understood, there is a greater likelihood, that the teacher will be able to respond to that challenge in a productive and supportive manner. We bring this theoretical understanding of the challenges to reflection together with Malinen's $(2000,2003)$ theorization of the role and responsibilities of an adult educator (as balancing the four dimensions of epistemic, existential, ethical and temporal), in order to conceptualize some essential features of a safe and accepting learning environment in relation to the individual process of reflection.

\section{What is the Purpose of Reflection?}

In order to understand the dynamics of facilitating reflection in education, it is helpful to examine its function from a broader, human life perspective. In Mezirow's (1991, 2000) theory of transformative learning, the process of reflection is presented in connection with two different but related directions (Mälkki \& Green, 2014). Firstly, reflection (or critical reflection) is seen as a vehicle to more valid knowledge. The ability to become aware and critically review paradigmatic assumptions is indispensable if general knowledge is to move forward. Likewise critical reflection can be directed towards that which one has personally learned pre-reflectively. In that case, reflection is seen as the means both toward understanding or knowing one's experience. According to Mezirow (2000), "[a] defining condition of being human is our urgent need to understand and order the meaning of our experience, to integrate it with what we know, in order to avoid the threat of chaos" (p. 3). Reflection enables us to 'know' what was previously 'lived'. Knowing what moves us enables us to intentionally orient towards a more authentic existence. Secondly, reflection can also serve as a method for maintaining one's sense of coherence and continuity of one's sense of self in spite of continually moving through multiple contexts as well as changing meaning 
perspectives. This result is more likely to occur when one identifies with one's sense of agency rather than one's meaning perspective (Mälkki \& Green, 2014). Thus, reflection may be seen to serve two intertwined directions, toward more truthful understanding of the world and knowledge, and toward more truthful understanding of one's own being and experience (Mälkki \& Green, 2014). Transformative learning as well as possibilities for creative and critical action may be seen to be enhanced by them both.

\section{Revisiting the Notion of 'Facilitating Reflection in Safe and Accepting Learning Environment'}

The idea that reflexivity requires a safe and accepting learning environment is an idea that is often voiced in educational literature. Specific practices for generating such an environment in the service of transformation have also been articulated (e.g. Mezirow, 1991; Cranton, 2006; Jokikokko, 2009). However, we wonder if the notion has become an 'empty' generalization that resonates with our intuition, but doesn't forward our theoretical understanding of the processes involved. In particular we claim that there is an "in-between" zone that partakes or joins both theory and practice. In the following we reflect on this notion itself, in order to form more detailed questions for analysis.

\subsection{Social Environment as Individually Experienced}

Often conceptions of reflection emphasize an intra-individual perspective without considering the relationship between individual processing and the social environment (see Fleming, 2014). However, our focus on the importance of the social and emotional environment in facilitating reflection implies that reflection requires more than a simple, intellectual reorganization. The cultural and institutional context and the relations among the participants as well as their relationship to the facilitator forms the context wherein reflection either takes place or doesn't. For example, a classroom in disrepair, with drug addicts loitering around the entrance, does not signal a safe environment. Likewise, a teacher who conveys the expectation that there is only one right answer will not encourage reflection but rather imitation and conformity.

However, the notion of safe and accepting learning environment does not just refer to the objective characteristics of an environment but to something that is individually experienced: (the expectation) that one feels oneself accepted and that one's contributions to the discussion will be received, even contemplated in a given social environment. Furthermore, because this environment is something that is individually experienced, through one's socially formed meaning perspectives (Mezirow, 1991), we cannot assume the environment to be or feel the same for everyone.

\section{2 "Facilitated"-A Facilitator, an Authority?}

Within adult education, facilitated dialogue is often considered as a means for promoting transformative learning. The facilitator initiates and guides the activities from an implicit position of authority that is different than that of the participants (see Cranton, 2006). Yet dialogue is frequently conceptualized as a conversation amongst equals rather than within hierarchies of authority and power (see Mezirow, 1991). Thus, to better understand the role of 
dialogue in facilitating transformative learning, it is necessary to examine the role of the facilitator, and its relationship to the participants. Specifically, we ask, how it is possible to reconcile the impact of an authority figure with the aims of dialogue (see Cranton, 2006; Rainio, 2010; Rainio \& Marjanovic-Shane, 2013; cf. Matusov, 2009)?

\section{3 "Facilitated"-Reflection on Demand?}

The claim that "a safe and accepting learning environment facilitates reflection" assumes that reflection can be facilitated. This assumption needs examination. Facilitation often comes with goals that, in the context of educational programs, may easily turn into expectations or demands, especially if it is tied to course gradings. In such a context, authority can, intentionally or unintentionally, continue to influence or condition a dialogue towards predetermined outcomes. However, earlier research indicates that reflection is something one cannot demand. This is particularly true if the educator is expecting reflection to deliver predetermined results (e.g. Mälkki \& Lindblom-Ylänne, 2012). That is to say, the more specific the expectation, the greater the likelihood that they will be fulfilled on the surface level, but at the expense of both the authenticity of the process and the actual transformative potential of reflection (Mälkki, 2011; Cranton, 2006). While it may be possible to "push" people to become aware of their assumptions, that does not necessarily result in revised assumptions that could generate new behaviors and understanding (Mälkki, 2011). In the end, the making of meaning is an act of personal commitment-on the existential level-and as such something that cannot be demanded (Malinen, 2000), in an ethically sound educational praxis. In facilitating reflection there must thus be a delicate understanding about the nature of reflection, so that the facilitation does not become disguised socialization.

\subsection{What is Non-Safe and Non-Accepting about the "Default" Learning Environment that Is} in Need of Adding Safety and Acceptance to It?

Addressing the need for safe and accepting learning environments implies that these condition are not always present while failing to specify what makes these setting unsafe. Articulating these less than ideal conditions would be the first step in remedying them. Both a starting point (actual conditions) and an end point (ideal outcomes) need to be articulated as a means for charting the path from "here" to "there".

\subsection{What Constitutes an Experience of a Safe and Accepting Learning Environment}

Above we noted that the notion of safe and accepting learning environment, in the end, becomes realized in the experience of an individual learner, rather than through reference to objectively measurable properties of the environment. This begs the question of what constitutes an experience of a safe and accepting learning context which would support reflection and transformation?

\subsection{Why Does Reflection Need to Be Facilitated}

As mentioned above, it is often taken for granted that any approach that emphasize safety and acceptance provides a more fruitful context for reflection and transformative learning. At the same time, however, this notion implies that reflection, by its very nature, is somehow 
difficult-otherwise there would be no need to facilitate it. Consequently, any attempt to delineate effective facilitation needs to address what makes reflection so difficult. Effective facilitation would, by definition, lessen or overcome those difficulties.

Within the light of the above we suggest that to track the dynamics of safe and accepting learning environment is a matter of

a) explicating the challenges or prerequisites of reflection (that do not get addressed in the default context);

b) explicating how the safe and accepting learning environment addresses these challenges through its understanding of the resistances to, and motivation for reflective thinking;

c) explicating how does the addressing of these challenges facilitate reflection?

In the following we address each of these questions in order to (begin to) conceptualize, from a relational and phenomenological perspective, the dynamics of safe and accepting learning environment in facilitating reflection and transformative learning.

\section{Reflection as Pushing Against the Current-Embracing the Counterwave}

In earlier work (Mälkki, 2010, 2011; Mälkki \& Green, 2014) we conceptualized the challenges and prerequisites of reflection by positioning it in the context of our human condition where both biological and social factors operate to insure our survival in the face of profound ontological and epistemological challenges. Here the challenges of engaging in reflection become visible in the negotiation between the intent to reflect, learn and develop, and the struggle to remain within the comfort zone by avoiding the very edge-emotions that paradoxically are the prime motivators for reflection (Mälkki, 2010, 2011). For example, if one has pre-reflectively learned that interpersonal conflict is dangerous and to be avoided, "because father becomes angry when mommy disagrees with him", then reflecting on one's conflict avoidant behavior is to surface the premise responsible for the behavior. Reflecting is also likely to evoke the fear that this premise was designed to avoid. However, without reflecting on that premise the person will employ it in contexts where the possibility of violence is minimal. The edge emotion of anxiety has the potential to enslave the person in a submissive, "nice guy", "good girl" style. In the following we will briefly explicate the theorization of reflection and edge-emotions, and elaborate on the prerequisites for safe and accepting learning environment.

The relationships that we will be exploring include that between reflection and edge-emotions, between individually experienced edge-emotions and comfort zone, and between edge-emotions and the collective comfort zone. Reflection is here understood in the manner that Mezirow (1991) used it: an effort to become aware of, and question the taken for granted assumptions governing one's thinking, feeling and acting (see Mälkki, 2011). The experience of edge-emotions and comfort zone occur as an interaction amongst the emotional, cognitive and social dimensions of experience (Mälkki, 2010, 2011). Thus, while these dimension refer to individual functioning, they open up a conceptual bridge to understanding the role of the social in learning, as well. As a result we can investigate the transformative potential of 
dialogue.

Comfort zone, as defined in our work (Mälkki, 2010, 2011), refers to the affective dimension of meaning perspectives. When we are able to carry on with our lives and interpret events, our social relations and ourselves unproblematically - according to our established meaning perspectives - we experience ourselves as sufficiently comfortable and safe. The world appears as understandable, and consequently we have confidence in our ability to survive. Although we may be aware of the possibility of multiple, alternate, interpretations we are able to maintain a sense of coherence and continuity as we apprehend the world via our expectations and previous understandings.

In contrast, when something questions our meaning perspectives, our taken-for-granted values or assumptions, we are thrown out of our comfort zones. We no longer experience ourselves as secure because uncomfortable edge-emotions have appeared. The authors have come to understand these emotions as a signal that our assumptions are being challenged and our meaning perspectives threatened (Mälkki, 2010, 2011). Thus, we may feel a number of unpleasant emotions including anxiety, fear, anger. We theorize that their appearance at the edges of one's comfort zone, relates to a threat to one's basic life-support system (Mälkki, 2010, 2011). As Damasio (1999) explains, emotions not only inform us of the suitability of our external or internal environment as being safe or dangerous, but also automatically orient us to action in order to avoid danger. Sometimes this orientation happens so quickly that we may not be conscious of the shift (Damasio, 1999). For example, a colleague rolls their eyes as we're making a presentation and the edge emotion thus evoked interferes with our remaining delivery. Our social acceptance has been threatened. Similarly, when our meaning perspectives, and thus our innate coherence-producing system is being threatened, our emotions mobilize us to restore our comfort zones (Mälkki, 2010, 2011).

On the positive side this kind of automatic orientation allows us to keep up our meaning perspectives and sense of coherence while avoiding the psychic fragmentation that can arise through both unrestricted or unimpeded reflection, and the loss of boundary that separates my point of view from yours. On the negative side this protective/defensive reaction can produce serious obstacles to learning, development and reflection. Thus, while the revelation that our meaning perspective is no longer adequate to life challenges can act as a stimulus for reflection (Mezirow, 1991), this very activation seems inhibited by unpleasant edge-emotions (Mälkki, 2010, 2011). While our assumptions may be seen to be in need of updating, they nevertheless have their history in being a part of our self, and bringing coherence to our understanding/being that we are reluctant to forgo (Mälkki \& Green, 2014). To let go of that meaning perspective brings with the threat of chaos that Mezirow so clearly articulated.

Why would we abandon assumptions that had worked so well in the past? Everything seems to be relatively stable as the present moment becomes assimilated to our past experiences, while simultaneously allowing us to form coherent expectations for the future. However, this does not go far enough toward explaining our resistance to reflection as it overlooks the influence of the social dimension. As Mezirow (1991) notes, our meaning perspectives are formed in social interaction, through culture and language. Thus, our meaning perspectives 
can be seen as individual compilations of shared social resources (Mälkki, Sjöblom, \& Lonka, 2014). Our personally held meanings are not only born out of, but also maintained by, social interaction. That is to say, shared meanings form connections between people bringing with them feelings of acceptance and recognition (Mälkki, 2011). It is these fragile bonds of belonging that our edge emotions are attempting to preserve. That is to say, to reflect on one's "personally held" assumptions, means risking the threat of exclusion (Mälkki \& Green, 2014). Brookfield's (1994) notion of cultural suicide is exemplary in this regard. Therefore, both our psychic need for coherency and our social need for belonging, govern when it is acceptable to question other's viewpoints or voice our own. This gives rise to a collective comfort zone (Mälkki, 2011), where all parties work to protect each other's comfort zones, and thereby preserve relationships. For much of the time this serves a positive purpose but on the negative side this may produce inhibited, conventional dialogue, when some fresh thinking is required by the circumstances being encountered. Clearly both our need for psychic coherency and social belonging are major factors to consider when we try to conceptualize both resistance to reflection and prerequisites for dialogue.

The edge-emotions operate as thresholds beyond which we leave our comfort zone. We can, and frequently do, avoid those emotions by holding, rather than examining, our assumptions. Instead we can 'explain' the problem away or blame the other, and thus disown how we are implicated. A common example takes the form of, "I did ' $\mathrm{X}$ ', because you did ' $\mathrm{Y}$ '" (Mälkki, 2010, 2011). This implies the premise that other people cause my behavior and therefore I am not morally culpable. In this way, our cognitive resources become channeled into service of maintaining comfort zone, rather than increasing understanding or critical self-awareness through reflection.

On the other hand, the edge-emotions can also be used as a prompt for reflection, as it is through these emotions that the problematic assumptions may be accessed and identified. This becomes more possible when we value the edge-emotions as a potential doorway to a greater existential truth. This construal of edge emotions as natural, normal and even worth welcoming stands in stark contrast to the general Western view that they are irrational and shameful. From our point of view, however, the path toward more rational thinking would go precisely through embracing the potential wisdom that these unpleasant emotions signify (Mälkki, 2010, 2011; Mälkki \& Green, 2014). These ideas are given a concrete form in the following aphorism, “you should be grateful whenever you're frustrated or disappointed because it means that you're getting news from reality". This adage acts as a reference point that radically changes our disposition toward the emergent from one of denial or repudiation to one of acknowledgement and even gratitude. When kept in mind, it allows the person to begin to identify their assumptions as, at least partially, distinguishable from the "news" of one's situation.

We preserve, rather than examine, our assumptions, because it keeps our meaning system and our identity stable. Secondly, it allows us to maintain the impression that those systems are adequate for any potential challenge that comes our way. Thirdly, it reinforces our sense of belonging with those with whom we share assumptions. Consequently, reflecting on those very assumptions, entails the risk of alienation from that group as well as loss of assumed 
adequacy. Although we have theoretically separated these challenges to reflection into the psychological and social, in practice it is often difficult to fully disentangle the intra-subjective from their social origins. Thus, a challenge to one of these dimensions impinges the others as well.

The above considerations reveal how reflection may be experienced as a "threat" to our psycho-social functioning. Therefore, in order to facilitate reflection, this threat should be addressed by an appropriate pedagogical practice.

\section{Facilitating Reflection-What Needs to Be Facilitated?}

To summarize, we have now recontextualized the challenges to reflection as natural in the sense that they are part of the system that maintains our psychic stability. It is not simply a matter of bypassing these resistances and proceeding directly to reflection. Rather, we must first valorize the pre-reflective intelligence of edge-emotions as the privileged means for identifying problematic assumptions. Once identified, they can be critically examined (Mälkki, 2011). Secondly, we can build a safe and accepting classroom culture that mitigates (without completely eliminating) the risk of exclusion and alienation. In the following section we will examine the latter in more detail.

\section{Facilitating Reflection-How?}

To address the question of how to address socially based resistances to reflection we turn to Malinen's (2003, see also 2000) typology of teacher responsibilities. According to Malinen (2003), there are four dimensions present in a pedagogical relationship: epistemic, existential, ethical and temporal. First, epistemic dimension refers to the aspect of teaching that traditionally has been the primary focus-for example, to organize and present epistemic content, to conceptualize phenomena, and to disclose the knowledge structures of the learners. Second, the existential dimension refers to a way of being, to the particular person that interacts with students through their teacher role. Whereas the epistemological dimension is often non-symmetrical - i.e. the teacher as assumed expert - on the existential dimension the teacher student relationship is symmetrical or equal. According to Malinen (2003), the existential dimension cannot be taught, but only demonstrated through being authentic which indirectly but powerfully influences the students. The third, temporal dimension refers to the responsibility to understand and scan the temporal trajectories within different intervening processes of learning, and to plan the activities accordingly. For example, opening up a new paradigmatic understanding of the world, a transformative learning process, may take years to navigate, or may never be fully complete, while learning some specific content may take only a minute given that it is easily graspable from within one's existing meaning frameworks. The time-frame varies also according to an individual, based on their previous experience and knowledge. The fourth, ethical dimension refers to the balancing among these intertwined dimensions, and to detect one's limitations in each (Malinen, 2000, 2003).

\subsection{Meeting at the Point of Imperfection}

As stated above Malinen (2000) suggests that at the epistemic dimension the teacher student relationship is asymmetric while at the same time cautioning that the teacher has an ethical 
responsibility to be aware of their limitations (Malinen, 2000, 2003). Regarding some topics, the teacher may be an expert when compared to the student. The distance between their levels of expertise is exaggerated if the authority role has an overlay of infallibility. However, with regard to the process of reflection the situation is a bit more complex. On a general level the teacher may be an expert of reflection, but regarding the contents and the private pathways of given student's reflection the teacher cannot be the expert. The teacher may understand the general features of the process and the most common obstacles, but it is impossible for the teacher to know the specific assumptions and specific pathways of meaning that would be most productive for a given student. This brings in view the limits of the teacher's abilities and forces the teacher to respect the student's ownership of their own process. Thus the teacher needs to respect the emergent nature of reflection-as an ongoing process rather than a completed product. It is in this incompleteness that the potential for feelings of vulnerability are the highest. Others are witnessing the student's groping for words to first express the assumption they've discovered and secondly to subject that assumption to critical scrutiny with the possibility of identifying its inadequacies. One of the authors has used the following metaphor to reassure students and clients about this process. "Insects are the only animal that wear their skeleton on the outside of their body. As such it also functions as armor. The only problem is that there is no room to grow within the confines of that armor. So they have to molt-discard their old forms in order to grow into their new potential. However, that leaves them without their armor for as long as it takes the new exo-skeleton to form. That is, they are vulnerable. In order to compensate for this loss, insects seek out hiding places that will keep them safe from predators." I then add, “As growing students you shouldn't have to hide. Instead you should be supported as you go through this metamorphosis.

While the teacher may use various metaphors to stimulate certain kind of processing, but the teacher may not know exactly which of them and in what ways they may or may not stimulate reflection for given student. Furthermore, while the teacher may be a theoretical expert on reflection, or even an expert in facilitating reflection, they may not be adept at their personal reflection. As the challenges to reflection are not to be overthrown but rather negotiated for the first time, every time (see Mälkki, 2011; Rainio \& Hilppö, 2016), it is not possible for anyone to be perfect at the process of reflection. Personal familiarity with the process of reflection reveals that it not easy, automatic or unchallenging and thus can cultivate patience and a supportive presence to students embarking on that journey. Teachers who practice reflection know first hand that being comfortable with not knowing is a prerequisite. Thus the teacher has to be willing to let the student engage in their own process, rather than "filling in" that vacuum with their supposed 'expert' knowledge (see also Mälkki \& Green, 2014).

Therefore, this position of incompleteness or not knowing can be helpful in facilitating reflection. It requires us to acknowledge that we are not perfectly rational creatures but rather a work in progress. From this viewpoint, a teacher in an authority position who is also aware of their uncertainty, may act as an encouragement for the student to own theirs. This allows the student to understand that it is not just them who is imperfect, but rather a common feature of being human. Here the teacher may indirectly teach (see Malinen, 2003), by being 
calm and confident in the face of uncertainty.

Reflection by its nature involves becoming aware of something that one used to hold as valid but now is being revealed as a) a taken for granted 'given', and b) possibly in need of revision. As such it requires encountering one's imperfections and inadequacies. Furthermore, if revision is required and new paths of meaning to be formed, it requires a leap of faith from the platform of one's previous assumptions into the clearing of the unknown (Mälkki \& Green, 2014). The experience of continuity and ontological security which had been anchored within previous understandings is being suspended and this may provoke questions of confidence in other meanings as well.

\subsection{Ethical Authority-Existential Collective Comfort Zone}

The above described challenges to reflection clearly point to a need for a facilitatorsomeone who can accompany the student as they leap toward a ground that is yet to be formed. The epistemic asymmetry that characterizes the position of authority may serve a purpose as it can act as a beacon that the student can trust when their internal landscape is in turbulence (see Mälkki \& Green, 2014). Authority can be exercised effectively by offering confirmation that the process of reflection and critical thinking is valid even though one cannot know beforehand where it will lead. That is to say, the expertise or competence that the teacher enacts may offer the student a reason to trust the process because the teacher does.

Earlier we referred to the notion of a collective comfort zone (Mälkki, 2011) regarding the implicit understanding of what, how and to what extent is it acceptable to express an opinion or question a teaching in a classroom. When the teacher-as a representative of the culture - holds explicit or implicit expectations for an appropriate outcome (see also Cranton, 2006) he or she can easily misuse the student's trust by encouraging conformity rather than critical thinking (see Malinen, 2003). However, this natural tendency to sense the comfort zones of the other, may also be utilized ethically in facilitating reflection. That is to say, the teacher may explicitly and momentarily suspend their membership in a community of like-minded individuals. And, further, they can surrender the effort of portraying oneself as exclusively rational. In addition, the teacher may question the implicit expectation to "sell" their viewpoint as the preferred outcome of education. Thus the teacher may decline offering reassurance through an appeal to shared assumptions. Rather, the teacher may offer the assurance that we all share the human condition-creatures moving toward their own mortality and responsible for their own meaning making. Although individuals have their own unique experiences and history, they have in common this existential condition. In this way, the collective comfort zone might not be exclusively anchored within the epistemic register - where the boundaries of acceptability are found. Rather, it could be cultivated within the existential dimension where experience is inclusive (as opposed to the exclusive nature of the epistemic). Thus their need to belong would be met through the reception and honouring of the student's own process of consulting their experience in the service of examining and revising their own assumptions. Thus belonging would not be bought at the expense of conformity. Rather, their cognitive and affective resources could be more freely employed to consider different viewpoints with the possibility, but not the demand, of 
adopting them for themselves.

In order to develop an existential collective comfort zone, it is vital for the teacher to be sensitive to the edges of their own comfort zone. For example, with regard to gradings, the teacher may explicate their own responsibility and personal challenges to be able to appreciate those student viewpoints that justifiably assess critically those viewpoints that the teacher has presented or applied in their practice. Here the teacher is in a way extending the edges of the epistemic collective comfort zone, which allows space for existential collective comfort zone to emerge. That is, the teacher explicitly addresses the common yet implicit assumption about the teacher valuing only those student papers that fall within the teacher's comfort zone. Furthermore, in focusing on facilitating safe and accepting learning environment, the teacher must be sensitive to the error of assuming that their comfort zone is also the collective's comfort zone.. Indeed, in many times in order to support the optimal learning environment for learners, per se, requires the teacher to work at the edges of their own comfort zones.

Existential collective comfort zone points to a phenomenological viewpoint of an optimal learning environment that respects humanness and free exploration of viewpoints; the teacher uses their power in the way that the innate need to feel accepted, becomes oriented away from epistemic conformity (epistemic collective comfort zone). Instead, the authority-supported, existential collective comfort zone allows one to use one's epistemic resources more freely, especially in relation to one's personal experiences. For example, if one were teaching the theory of transformative learning one could use relationship conflict with one's parents, children or partner to exemplify the emotional intensity and cognitive dissonance that occurs with disorienting dilemmas. Now the student has an experiential base to refer to when they are trying to integrate the abstractions of the theory. When one is not primarily attached to shared meanings but to the shared humanness, the exploration and revising of meanings appears less threatening. As teachers we cannot turn off the innate needs of the students, but we may have an effect on where they derive their satisfaction in our educational programs.

\subsection{The Peer Support for Diving into One's Own Experience}

Adult education traditionally involves more than the relationship between teacher and student because the peer group also exerts an influence on the collective comfort zone (Mälkki, 2011; see also Brookfield, 1994). Students are clearly attuned to the reactions of others to their offerings. This sensitivity to peer acceptance can take the form of excessive competitiveness and the fostering of alliances. Consequently it requires some ethical sensitivity from the teacher to channel this peer influence into a culture that fosters reflection. The teacher may, for example, explicitly acknowledge the fact that each individual is different, and thereby have their own unique experiences. This allows the student to feel sense of community with other unique individuals. Thus, acknowledgment of uniqueness may become the new social norm, which facilitates a fruitful framework for meeting the other in a dialogical space. The sharedness and commonness can be found on the existential level, whereas the epistemic level of opinions and viewpoints becomes positioned as if wobbly branches of a tree, 
something that need not be taken as static and overly definitive but rather as being in progress.

Here the social community may offer social confirmation and acceptance to this (new) approach and positioning within educational program - "because everyone else is doing so; it's not just me who's being carried away". The innate need for feeling accepted becomes related to the way of working rather than specific epistemic contents. This, in itself, may offer "compensation" for the stretching of social elastic bands, which an individual often experiences as threatening one's comfort zone and social relations.

\section{Conclusion}

In this paper we have explored the dynamics of facilitating reflection through a dialogic space of safe and accepting learning environment. We considered what makes reflection challenging in the first place, and based on that built conceptual framework to grasp some essential features of a classroom culture that supports reflection and critical thinking. We suggest that in order to understand the prerequisites of bringing about such a culture, it is fruitful for each of us to look into the ways that we strive to maintain our comfort zones to insure both our feeling of continuity and our sense of belonging. Thus, rather than just pushing towards the ideals, we need to understand what hinders us in the first place (Mälkki, 2011; Illeris, 2007). We come to understand that the apparent obstacles are actually the path to greater wisdom.

When our epistemic resources are not confined to protecting our comfort zones, and thus insulating us from experiencing, we suggest that the "urgent human need to ... understand one's experiences" (Mezirow, 2000) will have more fruitful environment in which to be realized. That is, the embodied, experiential feelings, images, ideas, felt senses (see Gendlin, 1997) may be more freely accessible to our conscious mind in the process of reflection, if our conscious mind is not preoccupied with reinforcing old meanings nor trying to remain within the collective comfort zone. Properly exercised ethical authority may offer the acceptance and encouragement for everyone to explore their own uniqueness. In this social situation this explorative process may produce the experience of sharedness, and the experience of this being 'normal' (see also West, 2014). Then, it depends on each individual what these circumstances, this safe and accepting learning environment, gives rise to. A necessary requirement for an ethical authority is their sensitiveness to the edges of their own comfort zones, so that the dialogical space of the class is not merely defined and bounded by the educator's edge-emotions.

In a metaphorical sense, the fertile ground for a plant to grow is formed with the initial guidelines: all points of view are welcome and need to be respected. The warmth is provided with the feeling of belonging and acceptance. The light refers to the insights that occur as a result of dialogue. In transformative learning it is the plant or the student that transforms, not the greenhouse. The teacher has an essential role in constructing the greenhouse, so that the student can grow into their unique, communicative self. However, often this takes the teacher into the process of transformative learning as well (Mälkki \& Green, 2014). 


\section{Acknowledgements}

The research is financed by Finnish Cultural Foundation. Thanks for PhD Anna Rainio, University of Helsinki.

\section{References}

Brookfield, S. D. (1994). Tales from the dark side: A phenomenography of adult critical reflection. International Journal of Lifelong Education, 13(3), 203-216. http://dx.doi.org/ $10.1080 / 0260137940130303$

Cranton, P. (2006). Understanding and Promoting Transformative Learning: A Guide for Educators of Adults. San Francisco: Jossey-Bass.

Damasio, A. R. (1999). The feeling of what happens. The Body and Emotion in the Making of Consciousness. New York: Hart Court Brace.

Freire, P., \& Shor, I. (1987). A pedagogy for liberation. Dialogues on transforming education (p. 80). Massachussets: Bergin \& Garvey. http://dx.doi.org/10.1007/978-1-349-18574-0

Gendlin, E. (1997). Experiencing and the Creation of Meaning: A Philosophical and Psychological Approach to the Subjective. Evanston: Northwestern University Press.

Hooks, B. (2007). Vapauttava kasvatus. Suomentanut Jyrki Vainonen. Helsinki; Kansanvalistusseura.

Illeris, K. (2007). How we learn. Learning and non-learning in school and beyond. London, England: Routledge.

Jokikokko, K. (2009). The role of significant others in the intercultural learning of teachers. Journal of Research in International Education, 8(2), 143-164. http://dx.doi.org/10.1177/147 5240909105202

Malinen, A. (2000). Towards the essence of adult experiential learning. A reading of the theories of Knowles, Kolb, Mezirow, Revans and Schön. Jyväskylä: Sophi, University of Jyväskylä.

Malinen, A. (2003). Opettajuus rakentuu ihmistuntijuudesta ja asiantuntijuudesta. In T. P. Sallinen, \& A. Malinen (Eds.), Opettajuus muutoksessa (pp. 63-92). Aikuiskasvatuksen 43. vuosikirja. Helsinki: KVS-Kirjat.

Mälkki, K. (2010). Building on Mezirow's theory of transformative learning: Theorizing the challenges to reflection. Journal of Transformative Education, 8, 42-62. http://dx.doi.org/ $10.1177 / 1541344611403315$

Mälkki, K. (2011). Theorizing the nature of reflection (Doctoral dissertation). University of Helsinki, Helsinki, Finland. Retrieved from https://helda.helsinki.fi/bitstream/handle/10138/ 26421/theorizi.pdf?sequence $=1$

Mälkki, K. (2012). Rethinking disorienting dilemmas within real-life crises: The role of reflection in negotiating emotionally chaotic experiences. Adult Education Quarterly, 62, 
207-229. http://dx.doi.org/10.1177/0741713611402047

Mälkki, K., \& Green, L. (2014). Navigational aids: The phenomenology of transformative learning. Journal of Transformative Education, 12, 5-24. http://dx.doi.org/10.1177/1541344 614541171

Mälkki, K., \& Lindblom-Ylänne, S. (2012). From reflection to action? Barriers and bridges between higher education teachers' thoughts and actions. Studies in Higher Education, 37, 33-50. http://dx.doi.org/10.1080/03075079.2010.492500

Mälkki, K., Sjöblom, K., \& Lonka, K. (2014). Transformation of the Physical Space and Transformation of the Subject. In A. Nicolaides, \& D. Holt (Eds.), Spaces of Transformation and Transformation of Space (pp. 550-556). Proceedings of the XI International Transformative Learning Conference, New York, Teachers College, Columbia University.

Matusov, E. (2009). Journey into dialogic pedagogy. New York: Nova Science.

Mezirow, J. (1991). Transformative dimensions of adult learning. San Francisco, CA: Oxford Jossey-Bass.

Mezirow, J. (2000). Learning to think like an adult. Core concepts of transformation theory. In J. Mezirow \& Associates (Eds.), Learning as transformation. Critical perspectives on a theory in progress (pp. 3-33). San Francisco, CA: Jossey-Bass.

Rainio, A. P. (2010). Lionhearts of the playworld: An ethnographic case study of the development of agency in play pedagogy (Doctoral Degree). Finland, Helsinki: University of Helsinki.

Rainio, A., \& Hilppö, J. (2016). The dialectics of agency in educational ethnography. Ethnography and Education, 1-17. http://dx.doi.org/10.1080/17457823.2016.1159971

Rainio, A. P., \& Marjanovic-Shane, A. (2013). From ambivalence to agency: Becoming an author, an actor and a hero in a drama workshop. Learning, Culture and Social Interaction, 2, 111-125. http://dx.doi.org/10.1016/j.lcsi.2013.04.001

West, L. (2014). Love, Actually, and Recognition, in Transformative Learning: Connecting the Individual and Collective in a Theory of Recognition. In A. Nicolaides, \& D. Holt (Eds.), Spaces of Transformation and Transformation of Space (pp. 114-118). Proceedings of the XI International Transformative Learning Conference, New York, Teachers College, Columbia University.

\section{Copyright Disclaimer}

Copyright for this article is retained by the author(s), with first publication rights granted to the journal.

This is an open-access article distributed under the terms and conditions of the Creative Commons Attribution license (http://creativecommons.org/licenses/by/3.0/). 